\title{
Short OGA is targeted to the mitochondria and regulates mitochondrial reactive oxygen species level
}

Patrick Pagesy $^{1}$, Abdelouhab Bouaboud ${ }^{1}$, Zhihao Feng $^{1}$, Philippe Hulin ${ }^{2}$ and Tarik Issad ${ }^{1}$

Running title: Short OGA is a mitochondria-targeted isoform

${ }^{1}$ Université de Paris, Institut Cochin, CNRS, INSERM, F-75014 Paris, France

${ }^{2}$ Université de Nantes, CHU Nantes, Inserm, CNRS, SFR Santé, Inserm UMS 016, CNRS UMS 3556, F-44000 Nantes, France

Address correspondence to:

Tarik Issad, Institut Cochin, Department of Endocrinology, Metabolism and Diabetes, 24 rue du Faubourg Saint-Jacques, 75014 Paris FRANCE. Tel: + 3314441 25 67;

E-mail: tarik.issad@inserm.fr 


\begin{abstract}
O-GlcNAcylation is a reversible post-translational modification involved the regulation of cytosolic, nuclear and mitochondrial proteins. Only two enzymes, OGT and OGA, control attachment and removal of O-GlcNAc on proteins, respectively. Whereas a variant OGT (mOGT) has been proposed as the main isoform that O-GlcNAcylates proteins in mitochondria, identification of a mitochondrial OGA has not been performed yet. Two splice variants of OGA (short and long isoforms) have been described previously. In this work, using cell fractionation experiments, we show that short-OGA is preferentially recovered in mitochondria-enriched fractions from HEK-293T cells as well as mouse embryonic fibroblasts. Moreover, fluorescent microscopy imaging confirmed that GFP-tagged shortOGA is addressed to mitochondria. In addition, using a BRET-based mitochondrial OGlcNAcylation biosensor, we show that co-transfection of short-OGA markedly reduced OGlcNAcylation of the biosensor, whereas long-OGA had no significant effect. Finally, using genetically encoded or chemical fluorescent mitochondrial probes, we showed that shortOGA overexpression increases mitochondrial ROS levels, whereas long-OGA had no significant effect. Together, our work reveals that the short-OGA isoform is targeted to the mitochondria where it regulates ROS homoeostasis.
\end{abstract}

Key words: O-linked N-Acetyl glucosaminylation, O-GlcNAcase, Mitochondria, Reactive Oxygen Species, Hydrogen peroxide, Oxidative stress 


\section{Introduction}

O-GlcNAcylation is a post-translational modification corresponding to the attachment of a single O-linked $\mathrm{N}$-acetylglucosamine (O-GlcNAc) to serine or threonine residues of cytosolic, nuclear or mitochondrial proteins. This reversible modification regulates the localization, the activity and the stability of proteins according to the nutritional environment of the cell, and more specifically according to glucose availability. Only two enzymes regulate O-GlcNAc level on proteins: OGT (O-linked N-Acetylglucosamine transferase), a glycosyl transferase that adds O-GlcNAc to proteins, and OGA, a $\beta-\mathrm{N}-$ Acetylglucosaminidase, distinct from acidic lysosomal hexosaminidase, which removes the O-GlcNAc from proteins. Numerous studies indicated a dynamic cross-talk between $\mathrm{O}-$ GlcNAcylation and phosphorylation, permitting fine-tuning of cell signalling pathways and regulation of gene expression (Issad \& Kuo, 2008; Issad et al, 2010). O-GlcNAcylation has been involved in important human pathologies, including neurogenerative diseases, diabetes and cancer (Bond \& Hanover, 2013).

Whereas protein O-GlcNAcylation in the cytosol and nucleus has been largely investigated, relatively little is known about O-GlcNAc cycling enzymes and their targets in the mitochondria. Alternative splicing of OGT results in the production of 3 different mRNA isoforms (Hanover et al, 2003; Love et al, 2003; Sacoman et al, 2017; Trapannone et al, 2016), that can code for 3 different proteins: a nucleo-cytoplasmic long form (ncOGT), a short isoform (sOGT) also found in the cytosol and nucleus, and at least in humans and nonhumans primates, a mitochondria-targeted variant (mOGT) (Supplementary figure 1). Several studies have shown the important role of O-GlcNAcylation in mitochondrial functions. Increases in O-GlcNAcylation of mitochondrial proteins have been observed upon highglucose conditions ( $\mathrm{Hu}$ et al, 2009; Makino et al, 2011), and recent work pointed to perturbation in the localisation of mOGT in cardiomyocytes mitochondria from diabetic mice (Banerjee et al, 2015). Several lines of evidence indicate that alteration of O-GlcNAc cycle disrupts mitochondrial homoeostasis (Akinbiyi et al, 2021; Jozwiak et al, 2021; Ma et al, 2015 ; Shin et al, 2011; Tan et al, 2017; Tan et al, 2014), including alteration in reactive oxygen species production (Jozwiak et al., 2021; Ngoh et al, 2011; Tan et al., 2017; Wang et al, 2016; Zhao et al, 2014).

Although OGA enzymatic activity has been demonstrated in mitochondria (Banerjee et al., 2015; $\mathrm{Hu}$ et al., 2009), the protein isoform involved has not been characterized. Alternative splicing of OGA also results in the production of two different mRNAs, coding for either long and short OGA isoforms (Kim et al, 2006). The long OGA comprise an O- 
GlcNAcase activity in its N-terminal side and pseudo histone acetyltransferase (HAT) domain in its C-terminal side (Hanover et al, 2012). In the human short OGA, the HAT domain is deleted, and a small intronic derived-sequence give rise to a unique 15 amino acids $\mathrm{C}$ terminal peptide. While the long OGA (L-OGA) isoform has been largely studied and was shown to be mainly cytoplasmic and nuclear, conflicting results have been reported concerning the short (S-OGA) isoform. Indeed, in glioblastoma cells, Comtesse et al. detected by western-blot a band of $130 \mathrm{kDa}$ corresponding to L-OGA in the cytosolic fraction, and a band of $75 \mathrm{kDa}$ in the nuclear fraction that they assumed to be S-OGA (Comtesse et al, 2001). In contrast, by fluorescence microscopy, Hanover's group did not detect GFP-tagged S-OGA in the nucleus but rather suggested a lipid-droplet localization for this protein in HeLa cells (Keembiyehetty et al, 2011).

Previous studies in cultured cells, animal tissues and human samples, have described a strong correlation between ncOGT and L-OGA expression (Kazemi et al, 2010; Pagesy et al, 2018; Slawson et al, 2005; Zhang et al, 2014), permitting tight control of O-GlcNAc level in the cell. While analysing OGT and OGA mRNA expression levels in human leukocytes from healthy donors, we noticed that in contrast to L-OGA mRNA which correlated with ncOGT mRNA, S-OGA mRNA expression did not correlate with ncOGT, but was tightly correlated with the mitochondrial mOGT mRNA (Supplementary Figure 1). This suggested a role for the short OGA isoform in the mitochondria and prompted us to evaluate its addressing to this organelle. We discovered that S-OGA is indeed the main OGA isoform in mitochondria and that it is involved in the control of ROS levels in this organelle.

\section{Results and discussion}

Long OGA has a theoretical molecular weight of $102 \mathrm{kDa}$ but is well known to run on SDS-PAGE with an apparent molecular weight of $130 \mathrm{kDa}$ (Gao et al, 2001). To evaluate the migration profile of short OGA on SDS-PAGE, we first transfected HEK-293T cells with pcDNA3 or with plasmids coding for either S-OGA or L-OGA. Proteins from total cell lysates were submitted to SDS-PAGE followed by western-blotting using an antibody directed against a region common to both isoforms (residues 500-550, Novus Biologicals antibody). As shown in Supplementary Figure 2A, transfected S-OGA and L-OGA were readily detected in total cell lysate. As expected, L-OGA has an apparent molecular weight of about $130 \mathrm{kDa}$ on SDS-PAGE. S-OGA, which has a theoretical molecular weight of $76 \mathrm{kDa}$, ran with an apparent molecular weight of about 95-100 kDa. Therefore, like L-OGA, S-OGA 
run on SDS-PAGE with an apparent molecular weight higher than predicted from its aminoacid sequence.

We then evaluated the relative distribution of endogenous L-OGA and S-OGA in total cell lysate (TCL), cytosolic (Cyto) and mitochondrial enriched (Mito) fractions from HEK293-T cells. In total cell lysates, we detected a major band of about $130 \mathrm{kDa}$ corresponding to the expected molecular weight of the long OGA, and fainter bands, including a band of about $95 \mathrm{kDa}$, possibly correponding to the short OGA isoform (Fig. 1A). Cell fractionation experiments indicated that L-OGA was mainly recovered in the cytosolic fraction, whereas SOGA was essentially recovered in the mitochondrial enriched fraction, although a band corresponding to L-OGA and an additional band of higher molecular weight were also detected in this fraction. Densitometric analysis of the blots indicated that the relative amount of S-OGA over L-OGA was much higher in the mitochondrial-enriched fraction (Fig. 1A and B).

Because antibodies quite often recognize non-specific bands that can be mistaken for the proteins of interest (Trapannone et al., 2016), we wanted to ensure that the $130 \mathrm{kDa}$ and 95 $\mathrm{kDa}$ bands detected by Novus anti-OGA antibody indeed corresponded to OGA by using cytosol and mitochondria enriched fractions of embryonic fibroblasts (MEF) from wild-type and OGA-KO mice (St Amand et al, 2018) (Fig. 1C). These two bands were detected in total cell lysates from wt-MEF, but not in OGA-KO MEF (left blot). An additional band of about $180 \mathrm{kDa}$ was also detected in total cell lysates, but it was present in both wt and OGA-KO $\mathrm{MEF}$, indicating that it corresponded to an unrelated protein recognized by this antibody. Cell fractionation of MEF showed that S-OGA was clearly the predominant form in the mitochondria-enriched fraction while L-OGA was predominant in the cytosol enriched fraction (Fig. 1C and D). These bands were undetectable in the corresponding fractions from OGA-KO MEF, confirming the identity of the bands and the good specificity of the antibody.

To further establish the cellular localization of S-OGA and L-OGA, we transfected GFPtagged version of these proteins in HEK-293T cells (Suppl. Figure 2B). In agreement with Keembiyehetty et al. (Keembiyehetty et al., 2011), transfected S-OGA-GFP and L-OGA-GFP were detected with an anti-GFP antibody as bands of about $130 \mathrm{kDa}$ and $160 \mathrm{kDa}$ respectively. As shown in Supplementary Figure 2B, L-OGA-GFP was more abundant than S-OGA-GFP in the cytosolic enriched fraction. In contrast, S-OGA-GFP was more abundant than L-OGA-GFP in the mitochondria-enriched fraction, despite higher expression of LOGA-GFP in total cell lysates, confirming the preferential expression of S-OGA in the mitochondria (Suppl. Fig. 2B, C). Similar results were obtained in MEF transfected with 
these constructs (Suppl. Fig. 2D, E), Thus, although S-OGA is clearly preferentially recovered in the mitochondrial-enriched fractions, both S-OGA and L-OGA isoforms were found in both compartments. Nonetheless, mitochondrial and cytosol-enriched fractions may have cross-contaminated each other during the centrifugation procedure, resulting in the recovery of S-OGA in the cytosolic fraction and L-OGA in the mitochondrial fraction. To confirm specific localization of S-OGA in the mitochondria, we used confocal microscopy. Since no antibodies are available to specifically detect the short OGA isoform by cell imaging, we co-transfected HEK-293T cells with cDNAs coding for either GFP-tagged short or long OGA isoforms and a mitochondrial-targeted mCherry protein. Confocal microscopy imaging (Fig. 2) revealed that L-OGA isoform was mainly found in the cytosol and the nucleus (Fig. 2A), whereas S-OGA was not detected in the nucleus (Fig. 2B), in agreement with Keembiyehetty et al observation (Keembiyehetty et al., 2011). In contrast, S-OGA isoform co-localized with mitochondrial mCherry protein (Fig. 2B), indicating specific mitochondrial addressing of this isoform. Mitochondrial localisation of the short OGA isoform was confirmed using structured illumination microscopy (Turkowyd et al, 2016)(Fig. 2C and D).

S-OGA results from an mRNA splicing that retains an intronic sequence coding for a 15 amino-acid peptide located in the C-terminus of the human short OGA isoform. This peptide sequence, which is not present in L-OGA, is fully conserved in five primate species and partially conserved in other mammalian species (Suppl. Fig.3). We hypothesized that this sequence may serve as a mitochondria-targeting sequence. To test this hypothesis, we introduced the sequence coding for this Intron-derived Peptide (IdP) at the C-terminus of the GFP. Transfection of HEK-293T cells with cDNA coding for either GFP or GFP-IdP showed that GFP-IdP was essentially recovered in the mitochondria enriched fraction, in contrast to GFP alone which was essentially recovered in the cytosolic fraction (Fig. $3 A$ and B). Confocal microscopy experiments confirmed mitochondrial localisation of IdP-GFP in HEK293 T as well as in HeLa cells (Fig. 3C). This result suggests that the intron-derived peptide may acts as a mitochondria-addressing sequence for S-OGA.

To determine whether S-OGA expression can regulate protein O-GlcNAcylation in mitochondria, we used a BRET-based O-GlcNAc biosensor that monitors O-GlcNAcylation activity in living cells (Al-Mukh et al, 2020; Groussaud et al, 2017). This biosensor comprises a lectin (GafD) domain fused to a luciferase and an O-GlcNAcylation substrate peptide derived from casein kinase 2 fused to a Venus fluorescent protein (Supplementary Fig. 4). O-GlcNAcylation of CKII promotes its binding to GafD, resulting in an increased in 
BRET signal (Al-Mukh et al., 2020), whereas removal of the GlcNAc by OGA will decrease BRET signal (Groussaud et al., 2017) (Suppl. Fig. 4). To specifically monitor OGlcNAcylation in mitochondria, the mitochondrial targeting sequence of cytochrome oxidase subunit 8A (COX8A) was fused the cDNA coding for the BRET-O-GlcNAc biosensor (Mitochondrial O-GlcNAc BRET biosensor, Suppl. Fig. 4). HEK293-T cells were cotransfected with this biosensor and either pcDNA3, S-OGA or L-OGA. We observed that cotransfection of S-OGA markedly reduced basal BRET, whereas L-OGA had no significant effect on BRET signal (Suppl. Fig. 4 and Fig. 4A). These results strongly suggest that SOGA is indeed an important regulator of protein O-GlcNAcylation level in the mitochondria.

Several studies have indicated that O-GlcNAcylation is involved in the regulation of oxidative stress (Chen et al, 2018). Since mitochondria is an important player in ROS production, we evaluated the effect of S-OGA overexpression on mitochondrial ROS levels in HEK-293T cells. Using a mitochondrial superoxide detection assay (MitoROS ${ }^{\mathrm{TM}} 580$ ), we observed that S-OGA overexpression in HEK-293T cells significantly increased mitochondrial ROS level, when compared to pcDNA transfected cells, whereas L-OGA had no significant effect (Fig. 4B).

In the mitochondria, ROS are readily converted into more stable and less toxic hydrogen peroxide $\mathrm{H}_{2} \mathrm{O}_{2}$ by superoxide dismutase (Candas \& Li, 2014). HEK-293T cells were cotransfected with pcDNA3, S-OGA or L-OGA, and a mitochondrial GFP-derived $\mathrm{H}_{2} \mathrm{O}_{2}$ biosensor (HyPer7, (Pak et al, 2020)), which permits to specifically monitor $\mathrm{H}_{2} \mathrm{O}_{2}$ level in the mitochondria. We observed that S-OGA expression resulted in a significant increase in $\mathrm{H}_{2} \mathrm{O}_{2}$ in the mitochondria, whereas L-OGA had no significant effect (Fig. 4C).

Together, our results indicate that S-OGA in the mitochondria regulates protein $\mathrm{O}$ GlcNAcylation, superoxide and hydrogen peroxide levels in mitochondria.

\section{Conclusion}

In this work, we discovered that S-OGA is preferentially targeted to the mitochondria, where it appears to modulate ROS levels. Mitochondria is believed to be the main source of cellular ROS. Whereas $\mathrm{H}_{2} \mathrm{O}_{2}$ can act as a signalling molecule in the cell, excess ROS production and elevated $\mathrm{H}_{2} \mathrm{O}_{2}$ levels have deleterious effects and are involved in several pathological conditions, including cancer, inflammatory diseases, type 2 diabetes, neurodegenerative diseases, and aging. The use of mitochondria-targeted small molecules has been proposed as a potential therapeutic strategy, most notably to specifically deliver antioxidants to this compartment (Oliver \& Reddy, 2019; Smith et al, 2011). The discovery 
that S-OGA is addressed to the mitochondria, and that it plays a role in the regulation of ROS levels in this organelle, may open new avenues for the development of molecules with potential therapeutic value. To this aim, it will be necessary to fully characterize S-OGA targets in the mitochondria, to elucidate its mechanism of action in the regulation of ROS production, and to develop molecules that will specifically target S-OGA activity and/or interaction with its mitochondrial protein partners. 


\section{Material and Methods}

Antibodies

Anti-OGA antibody (NBP2-32233) was from Novus Biologicals. Anti-ATP-5A antibody (15H4C4) was from Abcam. Anti-GFP antibody was from Roche (clones 7.1-13.1). Antihuman GAPDH (sc-47724) and anti-alpha Tubulin (sc-8035) antibodies were from Santa Cruz.

\section{Expression of $S$ - and L-OGA $m R N A$ in human leukocytes}

Human leukocytes were obtained from blood samples of healthy volunteers (age $44.7 \pm$ 1.7, 32 females, 35 males) from the French blood Agency (Etablissement Français du Sang, Ile-de-France, Site Trinité; Agreement number INSERM-EFS:18/EFS/030). For each individual, 5-10 $\mathrm{ml}$ of blood were collected in EDTA tubes. Leucocytes were isolated after red blood cell lysis in 3 volumes of RBC Lysis Buffer (Santa Cruz). Leucocytes were pelleted by centrifugation at $280 \mathrm{~g}$ during $5 \mathrm{~min}$. This procedure was repeated once or twice to eliminate residual red blood cells. The pellet was then washed in PBS, lysed in Trizol, and total RNA were isolated as described previously (Strobel et al, 1999). RT-qPCR were performed as described previously (Pagesy et al., 2018) using the primers indicated in Supplementary Table 1 .

\section{Preparation of cytosolic and mitochondrial-enriched fractions.}

HEK-293T cells were cultured as described previously (Blanquart et al, 2006). Preparation of mitochondrial-enriched fractions from HEK-293T cells was performed as described below. Briefly, HEK-293T cells were cultured to confluence in T-175 flasks. Cells from one T-175 flask were collected by trypsin digestion, washed in PBS and re-suspended in $1 \mathrm{ml}$ of ice-cold homogenization buffer containing $10 \mathrm{mM}$ Hepes, $\mathrm{pH}$ 7.4, $250 \mathrm{mM}$ sucrose, 1 $\mathrm{mM}$ AEBSF and $1 \mathrm{mg} / \mathrm{ml}$ of pepstatin, antipain, leupeptin and aprotinin. Cells were then homogenized on ice by flushes (30 up and down) through a $1 \mathrm{ml}$ syringue with a $22 \mathrm{G}$ needle. Homogenates were then centrifuged twice at $1000 \mathrm{~g}$ for $10 \mathrm{~min}$ to pellet and discard nuclei and large debris. Mitochondria were then pelleted by centrifugation at $11000 \mathrm{~g}$ during $10 \mathrm{~min}$, washed with $1 \mathrm{ml}$ homogenization buffer, centrifuged at $11000 \mathrm{~g}$ for $10 \mathrm{~min}$ and resuspend in the same buffer. The supernatant of the first $11000 \mathrm{~g}$ centrifugation, mainly containing the cytosolic fraction, and the mitochondrial-enriched pellet fraction, were then stored at $-80^{\circ} \mathrm{C}$ for subsequent analysis by western-blotting.

15-45 $\mu \mathrm{g}$ of proteins from either cytosolic or mitochondrial enriched fractions were submitted to western-blotting as described previously (Liu et al, 1998). Mitochondrial and cytosolic fractions were controlled using anti-ATP5A and anti-human GAPDH or anti-alpha 
tubulin antibodies.

\section{Confocal microscopy experiments.}

HEK-293T cells plated on polylysine-coated coverslips were transfected with cDNA (100 $\mathrm{ng} / 40000$ cells) coding for mitochondrial-targeted mCherry (mCherry-Mito-7, a gift from Michael Davidson (Addgene plasmid \# 55102), (Olenych et al, 2007)) and either GFP-tagged long or short OGA isoforms (gifts from John A. Hanover). 48h after transfection, cells were fixed with 4\% paraformaldehyde and stained with DAPI (4',6-diamidino-2-phenylindole) for visualisation of the nuclei. Coverslips were sealed with ProLong diamond anti-fade mounting media (ThermoFisher Scientific) and analysed by confocal microscopy. Confocal and Structured Illumination Microscopy (SIM) was performed on the MicroPICell Facility of the University of Nantes using an inversed confocal Nikon A1 microscope coupled to the super resolution N-SIM. Z stack of 0.12 microm were performed using a $100 \times$ oil-immersion lens with high NA (SR ApoTIRF 100×, oil, NA: 1.49, Nikon). Images were acquired using NIS 4.2 software.

HeLa cells plated on coverslips in a 6 well plate $\left(3 \times 10^{5}\right.$ cells/well) were transfected with $10 \mathrm{ng}$ of cDNA coding for GFP or GFP-IdP using lipofectamine 2000 (Thermofisher Scientific). Cells were labelled with 200nM MitoTracker (Thermofisher Scientific) during 45 $\min$ at $37^{\circ} \mathrm{C}$ and then fixed with $4 \%$ paraformaldehyde, stained with DAPI (4',6-diamidino-2phenylindole) for visualisation of the nuclei and analysed by confocal microscopy using an inverted microscope (Leica DMI6000, objective lens 100×).

\section{BRET experiments}

The coding sequence of mitochondrial targeting sequence from human COX8A was inserted upstream of the cDNA coding for the general O-GlcNAc-BRET biosensor described previously (Al-Mukh et al., 2020; Groussaud et al., 2017). HEK-293T cells were cotransfected with this mitochondrial O-GlcNAc-BRET biosensor and either pcDNA3, S-OGA or L-OGA plasmids. Cells transfected with luciferase alone and either pcDNA3, S-OGA or LOGA plasmids were used to correct for background signal. BRET experiments were then performed exactly as described previously (Lacasa et al, 2005) using the Tristar2 LB 942 (Berthold) plate reader. Briefly, cells were pre-incubated for $5 \mathrm{~min}$ in PBS in the presence of $5 \mu \mathrm{M}$ coelenterazine. Each measurement corresponded to the signal emitted by the whole population of cells present in a well. BRET signal was expressed in milliBRET Unit (mBU). The BRET unit has been defined previously as the ratio $530 \mathrm{~nm} / 485 \mathrm{~nm}$ obtained in cells expressing both luciferase and YFP, corrected by the ratio $530 \mathrm{~nm} / 485 \mathrm{~nm}$ obtained under the same experimental conditions in cells expressing only luciferase (Issad et al, 2002; Nouaille 
et al, 2006). In each experiment, BRET signal was the mean of at least 10 successive measurement performed every min during at least 10-15 min. In each experiment, the mean of at least 10 repeated BRET measurements in a given experimental condition (see supplementary Figure 4) was taken as the BRET value obtained in this experimental condition (Al-Mukh et al., 2020). Delta BRET corresponded to the difference in BRET signal measured in cells transfected with pcDNA3 and cells transfected with either S-OGA or LOGA.

\section{Determination of mitochondrial ROS using MitoROS probe}

Mitochondrial ROS level was assessed using a Mitochondrial Superoxide Assay Kit according to the manufacturer instructions (Abcam). Briefly, HEK293-T cells $\left(10^{5}\right.$ cells/well in 12 well plates) were transfected with 500ng of pcDNA3 or cDNA coding for S-OGA or LOGA using Lipofectamine 2000. 24h after transfection, cells were transferred into 96 well black microplate and cultured for an additional 24h. Cells were then incubated with the MitoROS $^{\mathrm{TM}} 580$ fluorescent probe $\left(100 \mu \mathrm{l}\right.$ of MitoROS ${ }^{\mathrm{TM}} 580$ stain working solution added in each well) for $1 \mathrm{~h}$ at $37^{\circ} \mathrm{C}$. Fluorescence emission at $590 \mathrm{~nm}$ was then measured after stimulation at $540 \mathrm{~nm}$ using a CLARIOstar (BMG) fluorimeter.

\section{Determination of mitochondrial $\mathrm{H} 2 \mathrm{O} 2$ using HyPer7 fluorescent probe}

HyPer7 (pCS2+MLS-HyPer7, a gift from Vsevolod Belousov, Addgene plasmid $\# 136470)$ ) is an ultrasensitive fluorescent ratiometric probe for detection of mitochondrial $\mathrm{H}_{2} \mathrm{O}_{2}$. This GFP probe has two excitation maxima at 400 and $499 \mathrm{~nm}$ and one emission peak centred at $516 \mathrm{~nm}$. Upon oxidation, excitation and absorption spectra of Hyper7 changes in a ratiometric way with a decrease at $400 \mathrm{~nm}$ and an increase of the $499 \mathrm{~nm}$ peak (Pak et al., 2020).

HEK293-T cells $\left(10^{5}\right.$ cells/well in 12 well plates) were co-transfected with 500ng of cDNA coding for HyPer7 and 500ng of pcDNA3 or cDNA coding for S-OGA or L-OGA using Lipofectamine 2000. 24h after transfection, cells were transferred into 96 well black microplate and cultured for an additional 24h. Culture medium was removed and the cells were washed with PBS and incubated in $100 \mu \mathrm{l}$ PBS for fluorescence determination. Fluorescence was measured at 515/40 nm after excitation at 390/22 nm and 485/15 nm using a LB942 Tristar2 Berthold fluorometer. After removing background fluorescence, the ratio of fluorescence emission after excitation at $485 \mathrm{~nm}$ to fluorescence emission after excitation at $390 \mathrm{~nm}$ was taken as relative measurement of mitochondrial $\mathrm{H}_{2} \mathrm{O}_{2}$ levels. Results were expressed as fold-change in 485/390 ratio induced by S-OGA or L-OGA transfection compared to pcDNA3 transfected cells. 
Statistical analysis. Statistical analyses were performed using PRISM software. Comparisons between groups were performed using Student's t test, or ANOVA followed by Dunnett's post-test for multiple comparison analysis. Correlations were performed using Pearson analysis. 


\section{Acknowledgments}

We thank John A. Hanover for the plasmids coding for GFP-tagged short and long OGA isoforms, and for generously providing us with wt and OGA-KO mouse embryonic fibroblasts.

We acknowledge the IBISA MicroPICell facility (Biogenouest), member of the national infrastructure France-Bioimaging supported by the French national research agency (ANR10-INBS-04) for confocal and SIM imaging of S-OGA-GFP.

\section{Authors contribution}

$\mathrm{PP}, \mathrm{AB}, \mathrm{ZF}$ researched data, analyzed the results, contributed to discussion and edited the manuscript. PH performed SIM imaging. TI conceived the experiments, analyzed the data and wrote the manuscript.

\section{Funding}

This work was supported by the INSERM, the CNRS and the FRM (Fondation pour la Recherche Médicale). Zhihao Feng is a recipient of a $\mathrm{PhD}$ fellowship from the Chinese Scholarship Council.

\section{Duality of Interest}

The authors declare to have no conflict of interest related to this work. 


\section{References}

Akinbiyi EO, Abramowitz LK, Bauer BL, Stoll MSK, Hoppel CL, Hsiao CP, Hanover JA, Mears JA (2021) Blocked O-GlcNAc cycling alters mitochondrial morphology, function, and mass. Sci Rep 11: 22106

Al-Mukh H, Baudoin L, Bouaboud A, Sanchez-Salgado JL, Maraqa N, Khair M, Pagesy P, Bismuth G, Niedergang F, Issad T (2020) Lipopolysaccharide Induces GFAT2 Expression to Promote O-Linked beta-N-Acetylglucosaminylation and Attenuate Inflammation in Macrophages. J Immunol 205: 2499-2510

Banerjee PS, Ma J, Hart GW (2015) Diabetes-associated dysregulation of O-GlcNAcylation in rat cardiac mitochondria. Proc Natl Acad Sci US A 112: 6050-6055

Blanquart C, Gonzalez-Yanes C, Issad T (2006) Monitoring the activation state of insulin/insulin-like growth factor-1 hybrid receptors using bioluminescence resonance energy transfer. Mol Pharmacol 70: 1802-1811

Bond MR, Hanover JA (2013) O-GlcNAc cycling: a link between metabolism and chronic disease. Annu Rev Nutr 33: 205-229

Candas D, Li JJ (2014) MnSOD in oxidative stress response-potential regulation via mitochondrial protein influx. Antioxid Redox Signal 20: 1599-1617

Chen PH, Chi JT, Boyce M (2018) Functional crosstalk among oxidative stress and OGlcNAc signaling pathways. Glycobiology 28: 556-564

Comtesse N, Maldener E, Meese E (2001) Identification of a nuclear variant of MGEA5, a cytoplasmic hyaluronidase and a beta-N-acetylglucosaminidase. Biochem Biophys Res Commun 283: 634-640

Gao Y, Wells L, Comer FI, Parker GJ, Hart GW (2001) Dynamic O-glycosylation of nuclear and cytosolic proteins: cloning and characterization of a neutral, cytosolic beta-Nacetylglucosaminidase from human brain. J Biol Chem 276: 9838-9845

Groussaud D, Khair M, Tollenaere AI, Waast L, Kuo MS, Mangeney M, Martella C, Fardini Y, Coste S, Souidi M et al (2017) Hijacking of the O-GlcNAcZYME complex by the HTLV-1 Tax oncoprotein facilitates viral transcription. PLoS Pathog 13: e1006518

Hanover JA, Krause MW, Love DC (2012) Bittersweet memories: linking metabolism to epigenetics through O-GlcNAcylation. Nat Rev Mol Cell Biol 13: 312-321

Hanover JA, Yu S, Lubas WB, Shin SH, Ragano-Caracciola M, Kochran J, Love DC (2003) Mitochondrial and nucleocytoplasmic isoforms of O-linked GlcNAc transferase encoded by a single mammalian gene. Arch Biochem Biophys 409: 287-297

Hu Y, Suarez J, Fricovsky E, Wang H, Scott BT, Trauger SA, Han W, Hu Y, Oyeleye MO, Dillmann WH (2009) Increased enzymatic O-GlcNAcylation of mitochondrial proteins impairs mitochondrial function in cardiac myocytes exposed to high glucose. J Biol Chem 284: 547-555

Issad T, Boute N, Pernet K (2002) A homogenous assay to monitor the activity of the insulin receptor using Bioluminescence Resonance Energy Transfer. Biochem Pharmacol 64: 813817

Issad T, Kuo M (2008) O-GlcNAc modification of transcription factors, glucose sensing and glucotoxicity. Trends Endocrinol Metab 19: 380-389

Issad T, Masson E, Pagesy P (2010) O-GlcNAc modification, insulin signaling and diabetic complications. Diabetes Metab 36: 423-435

Jozwiak P, Ciesielski P, Zakrzewski PK, Kozal K, Oracz J, Budryn G, Zyzelewicz D, Flament S, Vercoutter-Edouart AS, Bray F et al (2021) Mitochondrial O-GlcNAc Transferase Interacts with and Modifies Many Proteins and Its Up-Regulation Affects Mitochondrial Function and Cellular Energy Homeostasis. Cancers (Basel) 13 
Kazemi Z, Chang H, Haserodt S, McKen C, Zachara NE (2010) O-linked beta-Nacetylglucosamine (O-GlcNAc) regulates stress-induced heat shock protein expression in a GSK-3beta-dependent manner. J Biol Chem 285: 39096-39107

Keembiyehetty CN, Krzeslak A, Love DC, Hanover JA (2011) A lipid-droplet-targeted OGlcNAcase isoform is a key regulator of the proteasome. $J$ Cell Sci 124: 2851-2860

Kim EJ, Kang DO, Love DC, Hanover JA (2006) Enzymatic characterization of OGlcNAcase isoforms using a fluorogenic GlcNAc substrate. Carbohydr Res 341: 971-982

Lacasa D, Boute N, Issad T (2005) Interaction of the insulin receptor with the receptor-like protein tyrosine phosphatases PTPalpha and PTPepsilon in living cells. Mol Pharmacol 67: 1206-1213

Liu JF, Issad T, Chevet E, Ledoux D, Courty J, Caruelle JP, Barritault D, Crepin M, Bertin B (1998) Fibroblast growth factor-2 has opposite effects on human breast cancer MCF-7 cell growth depending on the activation level of the mitogen-activated protein kinase pathway. Eur J Biochem 258: 271-276

Love DC, Kochan J, Cathey RL, Shin SH, Hanover JA (2003) Mitochondrial and nucleocytoplasmic targeting of O-linked GlcNAc transferase. J Cell Sci 116: 647-654

Ma J, Liu T, Wei AC, Banerjee P, O'Rourke B, Hart GW (2015) O-GlcNAcomic Profiling Identifies Widespread O-Linked beta-N-Acetylglucosamine Modification (OGlcNAcylation) in Oxidative Phosphorylation System Regulating Cardiac Mitochondrial Function. J Biol Chem 290: 29141-29153

Makino A, Suarez J, Gawlowski T, Han W, Wang H, Scott BT, Dillmann WH (2011) Regulation of mitochondrial morphology and function by O-GlcNAcylation in neonatal cardiac myocytes. Am J Physiol Regul Integr Comp Physiol 300: R1296-1302

Ngoh GA, Watson LJ, Facundo HT, Jones SP (2011) Augmented O-GlcNAc signaling attenuates oxidative stress and calcium overload in cardiomyocytes. Amino Acids 40: 895911

Nouaille S, Blanquart C, Zilberfarb V, Boute N, Perdereau D, Roix J, Burnol AF, Issad T (2006) Interaction with Grb14 results in site-specific regulation of tyrosine phosphorylation of the insulin receptor. EMBO Rep 7: 512-518

Olenych SG, Claxton NS, Ottenberg GK, Davidson MW (2007) The fluorescent protein color palette. Curr Protoc Cell Biol Chapter 21: Unit 2125

Oliver DMA, Reddy PH (2019) Small molecules as therapeutic drugs for Alzheimer's disease. Mol Cell Neurosci 96: 47-62

Pagesy P, Tachet C, Mostefa-Kara A, Larger E, Issad T (2018) Increased OGA expression and activity in leukocytes from patients with diabetes: correlation with inflammation markers Exp Clin Endocrinol Diabetes doi: 10.1055/a-0596-7337.

Pak VV, Ezerina D, Lyublinskaya OG, Pedre B, Tyurin-Kuzmin PA, Mishina NM, Thauvin M, Young D, Wahni K, Martinez Gache SA et al (2020) Ultrasensitive Genetically Encoded Indicator for Hydrogen Peroxide Identifies Roles for the Oxidant in Cell Migration and Mitochondrial Function. Cell Metab 31: 642-653 e646

Sacoman JL, Dagda RY, Burnham-Marusich AR, Dagda RK, Berninsone PM (2017) Mitochondrial O-GlcNAc transferase (mOGT) regulates mitochondrial structure, function and survival in HeLa cells. J Biol Chem, doi: 101074/jbcM116726752 [Epub ahead of print]

Shin SH, Love DC, Hanover JA (2011) Elevated O-GlcNAc-dependent signaling through inducible mOGT expression selectively triggers apoptosis. Amino Acids 40: 885-893

Slawson C, Zachara NE, Vosseller K, Cheung WD, Lane MD, Hart GW (2005) Perturbations in O-linked beta-N-acetylglucosamine protein modification cause severe defects in mitotic progression and cytokinesis. J Biol Chem 280: 32944-32956 
Smith RA, Hartley RC, Murphy MP (2011) Mitochondria-targeted small molecule therapeutics and probes. Antioxid Redox Signal 15: 3021-3038

St Amand MM, Bond MR, Riedy J, Comly M, Shiloach J, Hanover JA (2018) A genetic model to study O-GlcNAc cycling in immortalized mouse embryonic fibroblasts. $J$ Biol Chem 293: 13673-13681

Strobel A, Siquier K, Zilberfarb V, Strosberg AD, Issad T (1999) Effect of thiazolidinediones on expression of UCP2 and adipocyte markers in human PAZ6 adipocytes. Diabetologia 42: $527-533$

Tan EP, McGreal SR, Graw S, Tessman R, Koppel SJ, Dhakal P, Zhang Z, Machacek M, Zachara NE, Koestler DC et al (2017) Sustained O-GlcNAcylation reprograms mitochondrial function to regulate energy metabolism. J Biol Chem 292: 14940-14962

Tan EP, Villar MT, E L, Lu J, Selfridge JE, Artigues A, Swerdlow RH, Slawson C (2014) Altering O-linked beta-N-acetylglucosamine cycling disrupts mitochondrial function. $J$ Biol Chem 289: 14719-14730

Trapannone R, Mariappa D, Ferenbach AT, van Aalten DM (2016) Nucleocytoplasmic human O-GlcNAc transferase is sufficient for O-GlcNAcylation of mitochondrial proteins. Biochem J 473: 1693-1702

Turkowyd B, Virant D, Endesfelder U (2016) From single molecules to life: microscopy at the nanoscale. Anal Bioanal Chem 408: 6885-6911

Wang X, Feng Z, Wang X, Yang L, Han S, Cao K, Xu J, Zhao L, Zhang Y, Liu J (2016) OGlcNAcase deficiency suppresses skeletal myogenesis and insulin sensitivity in mice through the modulation of mitochondrial homeostasis. Diabetologia 59: 1287-1296

Zhang Z, Tan EP, VandenHull NJ, Peterson KR, Slawson C (2014) O-GlcNAcase Expression is Sensitive to Changes in O-GlcNAc Homeostasis. Front Endocrinol (Lausanne) 5: 206

Zhao L, Feng Z, Zou X, Cao K, Xu J, Liu J (2014) Aging leads to elevation of OGlcNAcylation and disruption of mitochondrial homeostasis in retina. Oxid Med Cell Longev 2014: 425705 


\section{Figure legends}

Figure 1: Detection of long and short OGA isoforms in HEK-293T cells and mouse embryonic fibroblasts (MEF).

(A) Detection of endogenous S-OGA (95 kDa) and L-OGA (130 kDa) bands in total cell lysates (TCL), mitochondrial enriched (Mito) and cytosolic enriched (Cyto) fractions from HEK-293T cells by western-blotting with anti-OGA Novus antibody. Mitochondrial and cytosol fractions were controlled using anti-ATP5A and anti-GAPDH antibodies. The $95 \mathrm{kDa}$ band was essentially recovered in the mitochondria-enriched fraction, whereas the $130 \mathrm{kDA}$ band was mostly recovered in the cytosol-enriched fraction.

(B) Densitometric analysis of the $95 \mathrm{kDa}$ and $130 \mathrm{kDa}$ OGA bands in HEK-293T cells. The results are the mean \pm SEM of the ratio of the $95 \mathrm{kDa}$ to the $130 \mathrm{kDa}$ OGA bands detected in cytosol- and mitochondria-enriched fractions ( $\mathrm{n}=9 ; * *: \mathrm{p}<0.01)$.

(C) Detection of endogenous S-OGA (95 kDa) and L-OGA (130 kDa) bands in total cell lysates (TCL), mitochondria-enriched (Mito) and cytosol-enriched (Cyto) fractions from wildtype (wt) and OGA-KO mouse embryonic fibroblasts (MEF) by western-blotting with antiOGA Novus antibody. Mitochondrial and cytosol fractions were controlled using anti-ATP5A and anti-tubulin antibodies. The $95 \mathrm{kDa}$ band was predominant in the mitochondria-enriched fraction, whereas the $130 \mathrm{kDa}$ band was predominant in the cytosol-enriched fraction of wtMEF. Both bands were absent in the corresponding fractions in OGA-KO MEF. An additional band, (about $180 \mathrm{kDa}$ ) was also detected in total cell lysates, but this band was present in both wt and OGA-KO MEF, indicating that it corresponded to an unrelated protein. (D) Densitometric analysis of the $95 \mathrm{kDa}$ and $130 \mathrm{kDa}$ OGA bands in wt-MEF. The results are the mean \pm SEM of the ratio of the $95 \mathrm{kDa}$ to $130 \mathrm{kDa}$ OGA signals detected in cytosoland mitochondria-enriched fractions $(n=6 ; *: p<0.05)$.

\section{Figure 2: Detection of GFP-tagged long and short OGA isoforms in HEK-293T cells.}

HEK-293T cells were transfected with mitochondrial-targeted mCherry and either GFPtagged short or long OGA isoforms, fixed, stained with DAPI for visualisation of the nuclei (blue, panels 1), and analysed by confocal microscopy in the GFP (green, panels 2) and mCherry (red, panels 3) channels. Merge images are shown in panels 4. (A) the long OGA isoform was detected in the cytosol and the nucleus but poorly co-localized with mito-cherry labelled mitochondria. (B) Co-localization of the short OGA isoform with mito-mCherry in a cell expressing GFP-tagged short OGA. (C, D) Structured illumination microscopy (SIM) 
confirmed localisation of S-OGA in the mitochondria. (C) Separated SIM and merge images of this cell in the GFP, mCherry and Dapi channels are shown. (D) Densitometric analysis of the different signals detected along the arrow shown on the upper panel indicates colocalisation of S-OGA-GFP with mito-mCherry (green: S-OGA-GFP, red: mito-mCherry, blue: Dapi staining of the nucleus, white bar scale: $5 \mu \mathrm{m})$. Representative images are shown.

Figure 3: The intron-derived peptide of short OGA is sufficient to target GFP to the mitochondria.

(A) HEK-293T cells were transfected with cDNA coding for either GFP or GFP fused at its C-terminus with OGA intron-derived peptide (GFP-IdP). Total cell lysate, mitochondrial and cytosol enriched fractions were analysed by western-blotting with anti-GFP antibody. GFPIdP was more abundant in the mitochondrial-enriched fraction whereas GFP was more abundant in the cytosol enriched fraction.

(B) Densitometric analysis of the GFP and GFP-IdP bands. The results are the mean \pm SEM of the ratio of the GFP-IdP to GFP signals detected in cytosol- and mitochondria-enriched fractions $(\mathrm{n}=5 ; *: \mathrm{p}<0.05)$.

(C) HEK-293T cells and HeLa cells were transfected with cDNA coding GFP fused at its Cterminus with OGA intron-derived peptide (GFP-IdP), fixed and labelled with mitotracker. Confocal microscopy experiments confirmed co-localisation of GFP-IdP with mitotracker. GFP alone was widely distributed into the cells, including the nucleus (HeLa cells).

Figure 4: Short OGA expression reduced O-GIcNAcylation and increase ROS levels in the mitochondria

(A) HEK-293T cells were co-transfected with the mitochondria-targeted BRET biosensor and either pcDNA3, S-OGA or L-OGA. The graph shows the difference in BRET signal (delta BRET, expressed in milliBRET Units) between pcDNA3 transfected cells and either S-OGA or L-OGA transfected cells. Data are the mean \pm SEM of 9 independent BRET experiments. Statistical analysis was performed using ANOVA followed by Dunnett's post-test (****: $\mathrm{p}<0.0001$, when compared to the control condition, NS: non-significative). S-OGA markedly reduced BRET signal, indicating decreased O-GlcNAcylation of the mitochondrial O-GlcNAc biosensor by this isoform.

(B) HEK-293T cells transfected with pcDNA3, S-OGA or L-OGA were incubated for $1 \mathrm{~h}$ with MitoROS $^{\mathrm{TM}} 580$. Fluorescence emission at $590 \mathrm{~nm}$ was then measured after excitation at 540 
$\mathrm{nm}$. Results are expressed as fluorescence in S-OGA or L-OGA transfected cells relative to pcDNA3 transfected cells, and are the mean \pm SEM of 5 independent experiments.

(C) HEK-293T cells were co-transfected with the mitochondria $\mathrm{H}_{2} \mathrm{O}_{2}$ biosensor (HyPer7) and either pcDNA3, S-OGA or L-OGA. In each experiment, the ratio of fluorescence emission at $516 \mathrm{~nm}$ after excitation at $499 \mathrm{~nm}$ to fluorescence emission at $516 \mathrm{~nm}$ after excitation at 400 $\mathrm{nm}$ was determined. Results are expressed as fold-change in 485/390 ratio in S-OGA or LOGA transfected cells compared to pcDNA3 transfected cells, and are the mean \pm SEM of 13 independent experiments. 


\section{Supplementary Figure 1: Correlations between S-OGA and mOGT mRNA expression} in human leukocytes (A) Alternative splicing of OGA results in the production of two different mRNAs, coding for either long or short OGA isoforms. The short mRNA isoform lacks the sequence coding for exons 11 to 16 but retains part of intron 10 as a coding sequence. The black box indicates the localization of the sequence that is specific for short OGA. 2 couples of primers were designed to specifically quantify mRNA expression of each isoform. (B) Alternative splicing of OGT results in the production of three different mRNAs, which can code for 3 different proteins: the two nucleo-cytoplasmic long (ncOGT) and short OGT variants (sOGT), and at least in human and other primates, a mitochondria-targeted variant (mOGT). The mOGT mRNA isoform is generated by the use of intron 4 as an alternative exon (exon 5). This transcript contains a unique ATG that produces a shorter isoform which comprises a 20 amino acid mitochondrial targeting sequence. 2 couples of primers were designed to specifically quantify mRNA expression of ncOGT and mOGT isoforms (the absence of any sequence specific to sOGT impairs evaluation of its expression).

(C) Correlations between OGT and OGA mRNA splice variants were evaluated using Pearson's analysis. OGA mRNA expression levels were measured in leucocytes from healthy donors by quantitative RT-PCR and normalized to the expression of cyclophilin A mRNA. Expression levels of ncOGT correlated with long OGA mRNA (left panel) but not with short OGA mRNA (middle panel), while mOGT mRNA expression levels correlated with short OGA (right panel).

\section{Supplementary Figure 2: Expression of transfected S-OGA and L-OGA in HEK-293 T and mouse embryonic fibroblasts}

(A) HEK-293T cells were transfected with pcDNA3 empty vector or plasmids coding for SOGA or L-OGA. S-OGA and L-OGA in total cell lysates were detected as bands of apparent molecular weight of $95 \mathrm{kDa}$ and $130 \mathrm{kDa}$ by western-blotting using anti-OGA Novus antibody.

(B) HEK-293T cells were transfected with plasmids coding for GFP-tagged S-OGA or LOGA. GFP-S-OGA and GFP-L-OGA (left panel) were detected as bands of apparent molecular weights of $130 \mathrm{kDa}$ and $160 \mathrm{kDa}$ by western-blotting with an anti-GFP antibody. In mitochondria-enriched fractions from these cells, recovery of transfected GFP-S-OGA was higher than GFP-L-OGA, whereas GFP-L-OGA recovery was higher in the cytosolic enriched 
fraction. Mitochondrial and cytosol fractions were controlled using anti-ATP5A and antiGAPDH antibodies.

(C) Densitometric analysis of the $130 \mathrm{kDa}$ and $160 \mathrm{kDa}$ GFP-OGA bands in HEK-293T cells. The results are the mean \pm SEM of the ratio of the $130 \mathrm{kDa}$ to $160 \mathrm{kDa}$ GFP-OGA bands detected in cytosol- and mitochondria-enriched fractions $(n=3 ; *: p<0.05)$.

(D) MEF were transfected with cDNA coding for either GFP alone, GFP-S-OGA (130 kDa) or GFP-L-OGA (160 kDa). (A) Total cell lysate (TCL), mitochondria (Mito) and cytosolic (Cyto) enriched fractions from these cells were submitted to western-blotting using an antiGFP antibody. Mitochondrial and cytosol enrichment was controlled using anti-ATP5A and anti- $\alpha$-tubulin antibodies. In mitochondria-enriched fractions, recovery of transfected GFP-SOGA was higher than GFP-L-OGA, whereas GFP-L-OGA recovery was higher in the cytosolic enriched fraction. (E) Densitometric analysis of the $130 \mathrm{kDa}$ and $160 \mathrm{kDa}$ GFPOGA bands in HEK-293T cells. The results are the mean \pm SEM of the ratio of the $130 \mathrm{kDa}$ to $160 \mathrm{kDa}$ GFP-OGA signals detected in TCL, cytosol- and mitochondria-enriched fractions $(\mathrm{n}=3 ; *: \mathrm{p}<0.05)$.

\section{Supplementary Figure 3}

Alignment of the sequences of short OGA intron-derived peptide in different mammalian species.

\section{Supplementary Figure 4}

(A) The O-GlcNAc BRET biosensor is composed of Rluc8 luciferase fused to a lectin domain (GafD), a known OGT substrate peptide derived from casein kinase II, followed by the Venus variant of the yellow fluorescent protein. The human COX8A (Cytochrome Oxydase subunit 8A) pre-sequence was inserted upstream of this biosensor for specific targeting to the mitochondria. Basal BRET signal results from the balance between OGT and OGA activities. Upon de-GlcNAcylation by OGA, decreased binding of casein kinase II peptide to GafD lectin domain results in a decrease in BRET signal. (B) Typical experiment showing the monitoring of BRET signal during 10 minutes in pcDNA3, S-OGA and L-OGA transfected HEK-293-T cells. BRET signal was markedly decreased by S-OGA but barely affected by LOGA. 
Supplementary Table 1: Sequences of oligonucleotides used for RT-qPCR

\begin{tabular}{|l|l|}
\hline long OGA Forward & ATGATGGCTTGCCTCCTGTC \\
\hline long OGA Reverse & CAACAAATGTCACAGGCTCCG \\
\hline short OGA Forward & GAGCATGGACCCAAAGGAGC \\
\hline short OGA Reverse & AGATTATTCCTGGTGCACCTACC \\
\hline ncOGT Forward & GCCCTGGGTCGCTTGGAAGA \\
\hline ncOGT Reverse & TGCCACAGCTCTGTCAAAAA \\
\hline mOGT Forward & AACCCCTCCTAAGGCATGTT \\
\hline mOGT Reverse & CACCACTGCGTGATTTGGACT \\
\hline Cyclophilin Forward & GGTGACTTCACACGCCATAATG \\
\hline Cyclophilin Reverse & ACAAGATGCCAGGACCCGTAT \\
\hline
\end{tabular}




\section{Figure 1}

\section{HEK-293T}

A

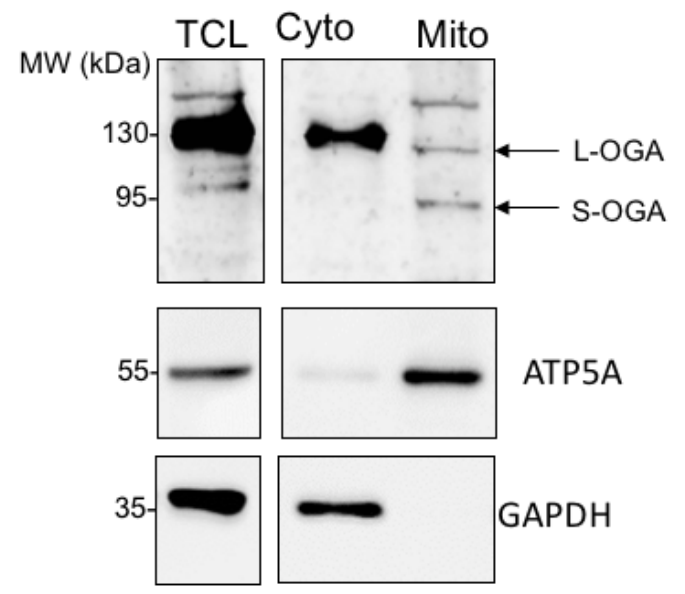

\section{MEF}

\section{C}

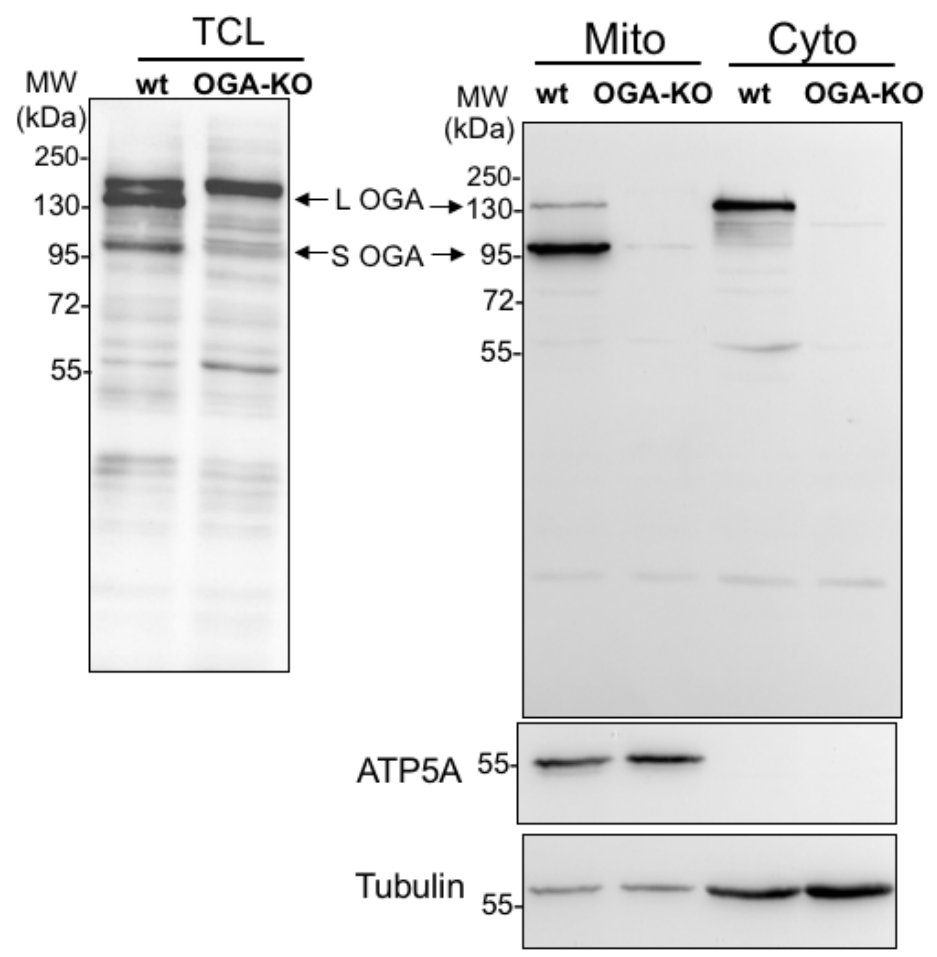

B

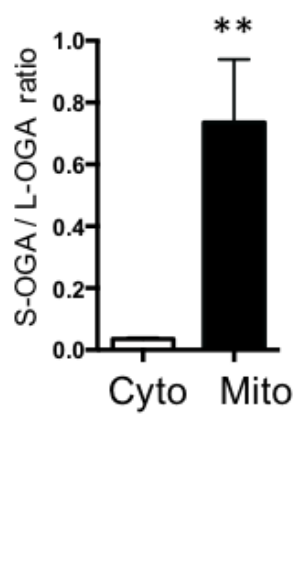

D

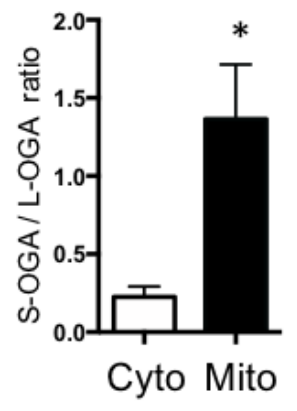


bioRxiv preprint doi: https://doi.org/10.1101/2021.12.25.474160; this version posted December 25, 2021. The copyright holder for this

preprint (which was not certified by peer review) is the author/funder. All rights reserved. No reuse allowed without permission.

Figure 2

A

Long OGA confocal

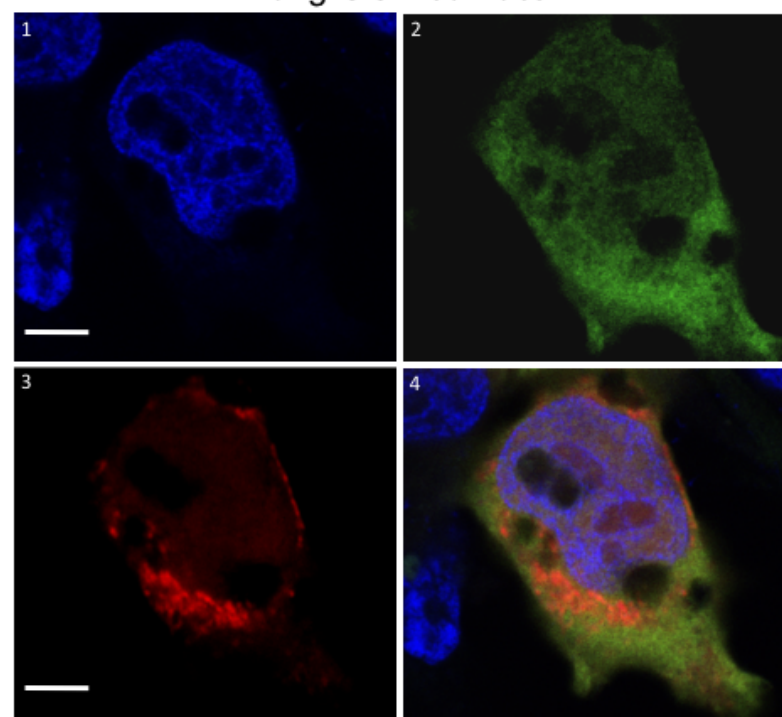

C

Short OGA SIM

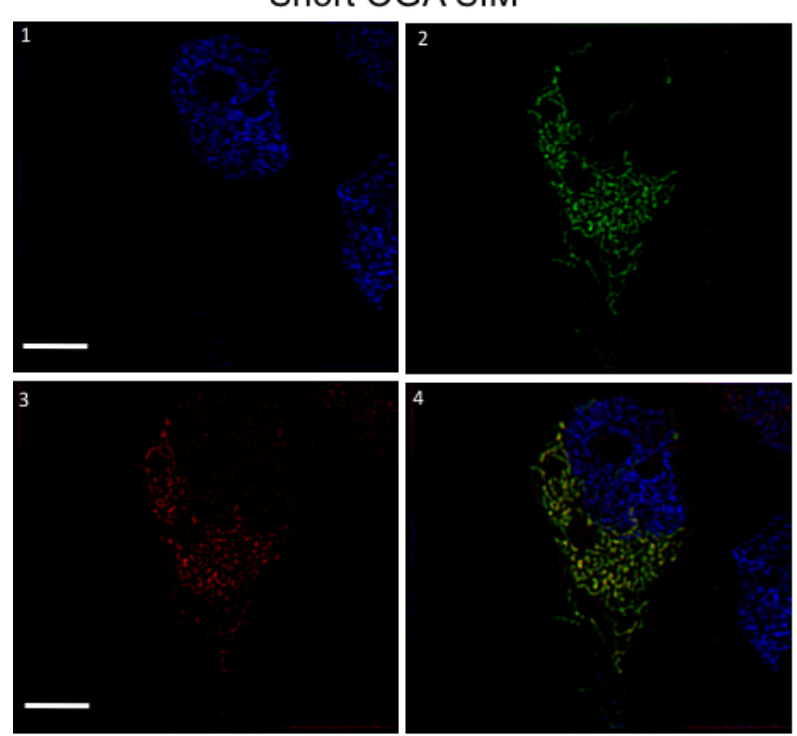

B

Short OGA confocal

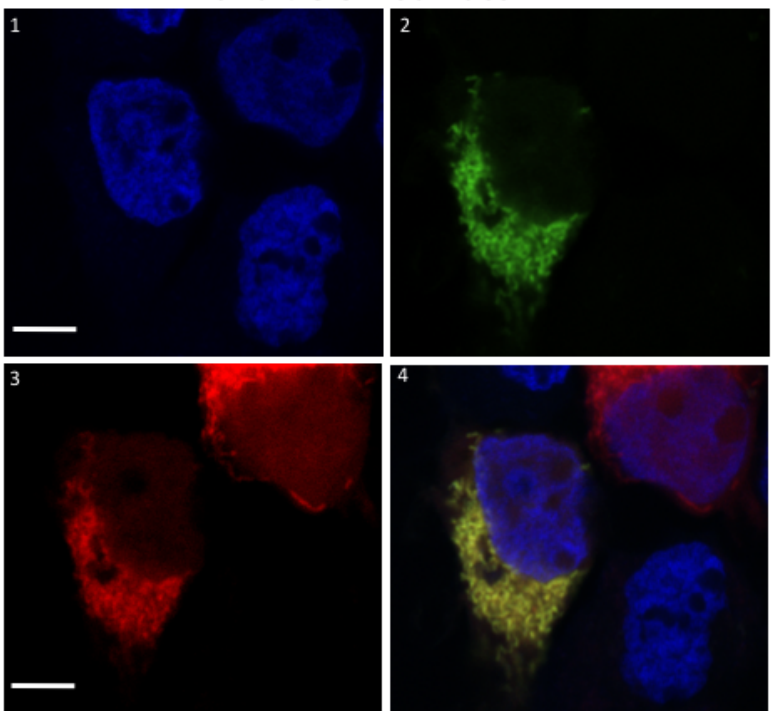

\section{Short OGA SIM}

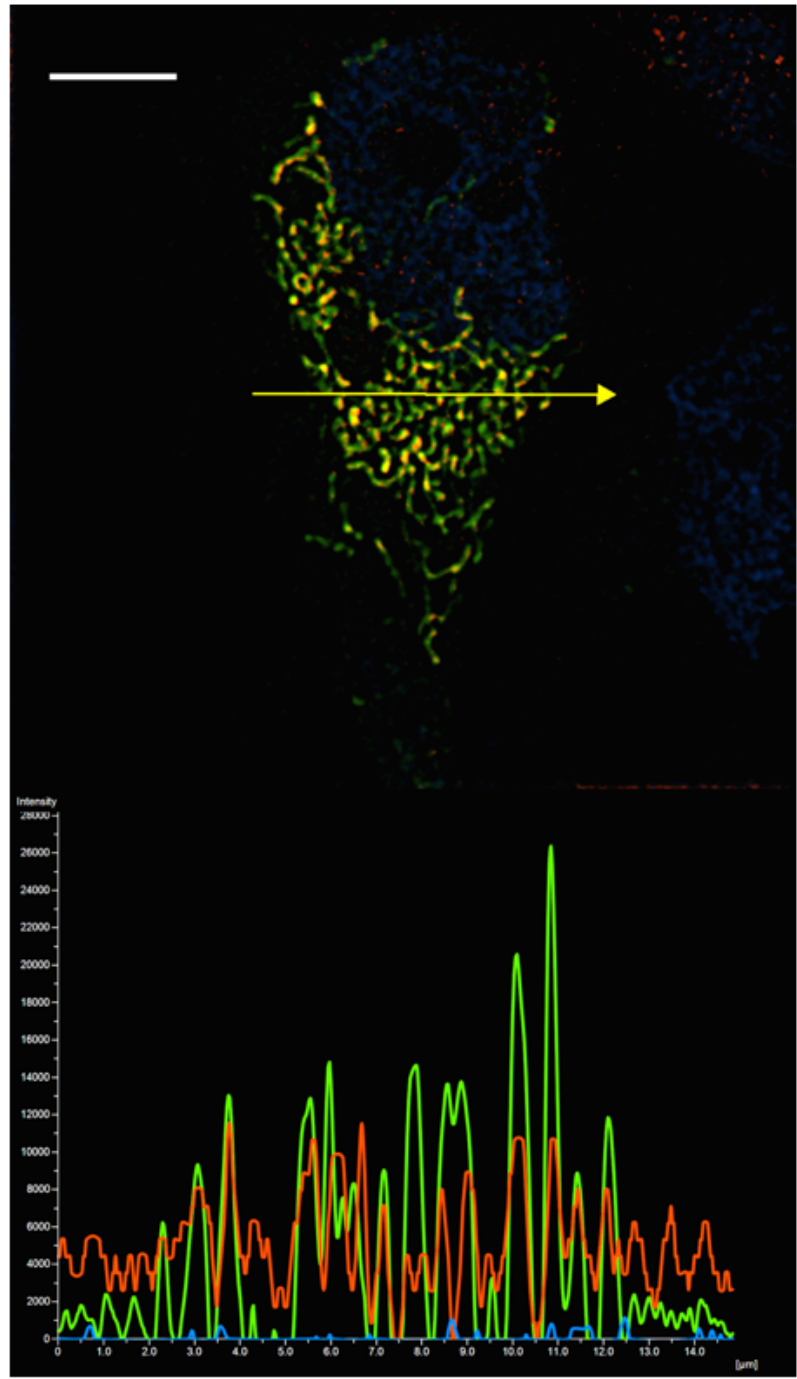


Figure $3^{\text {p }}$

A

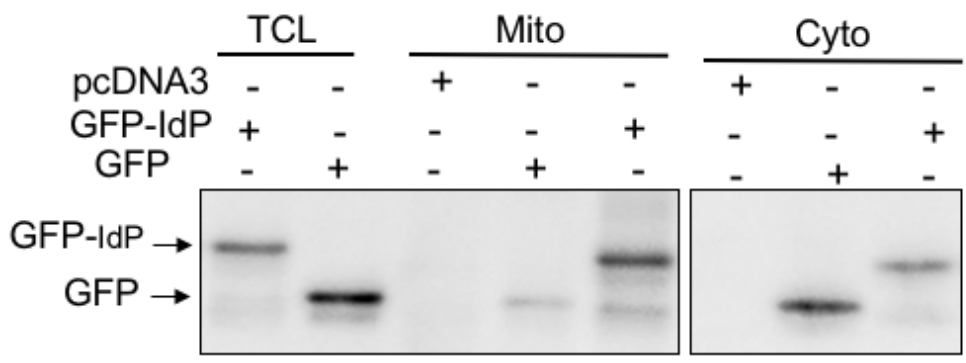

B
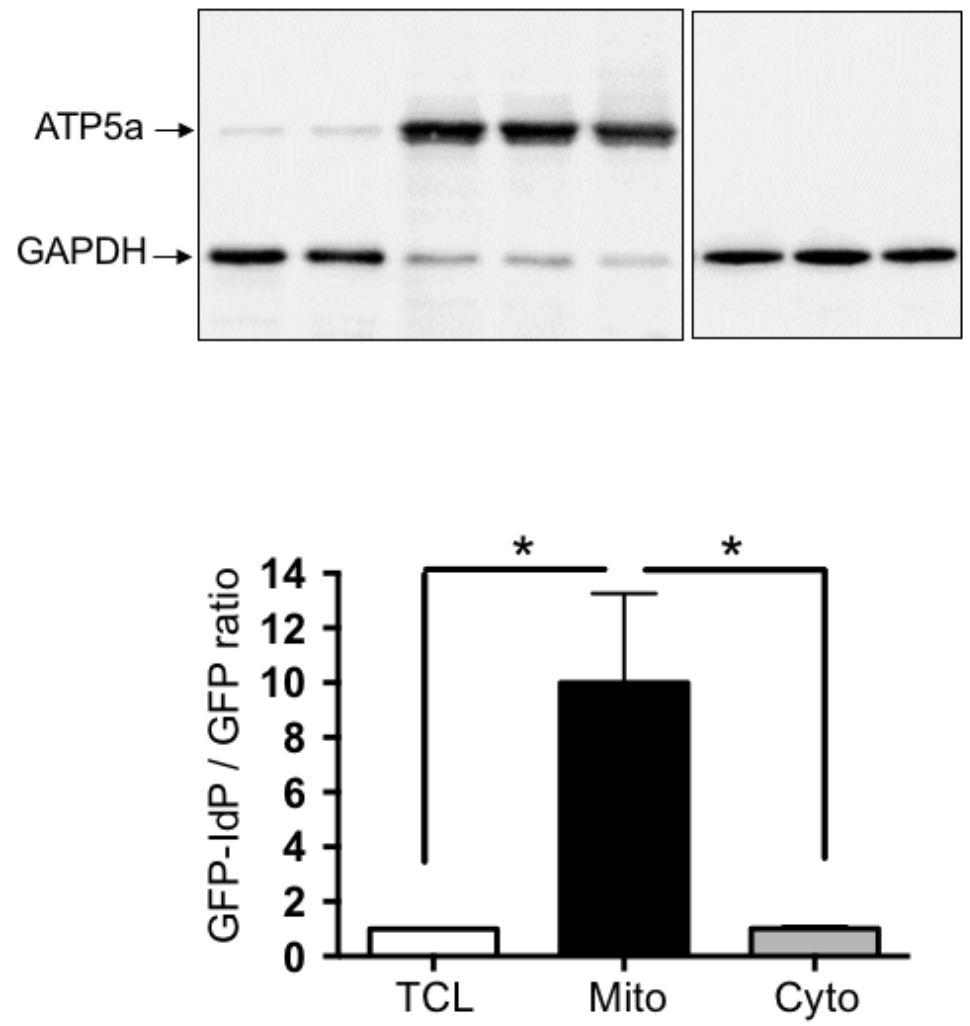

C
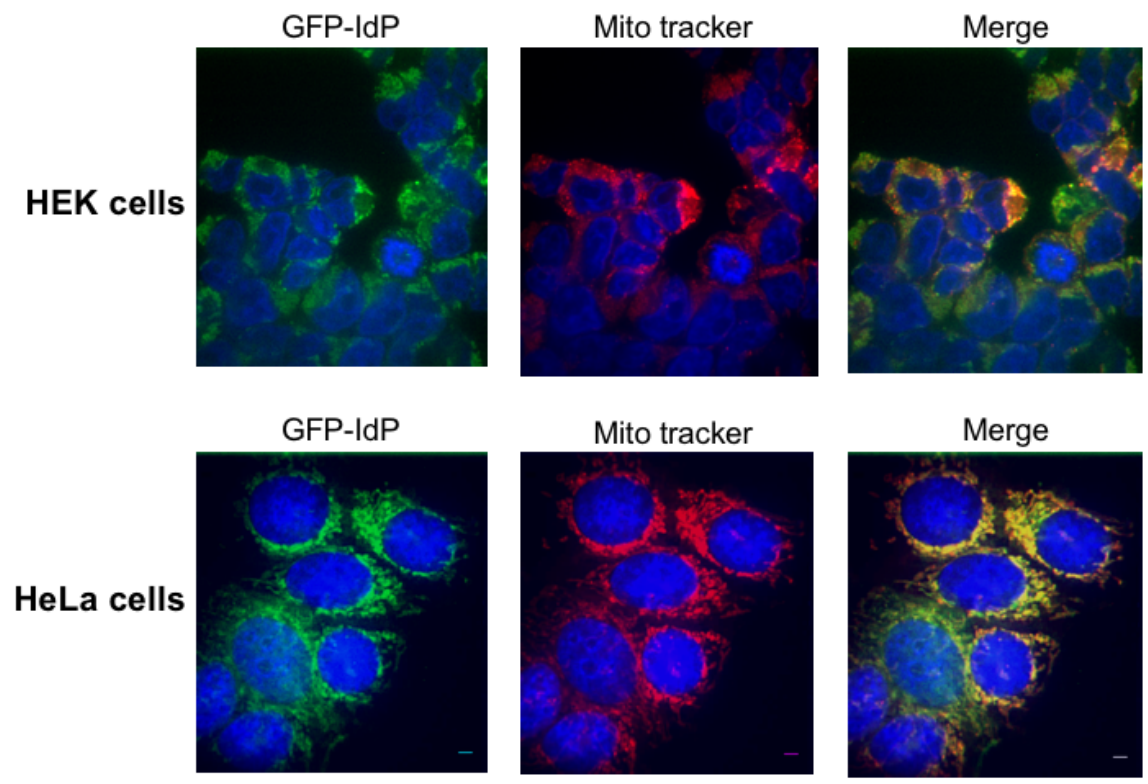

GFP

Mito tracker
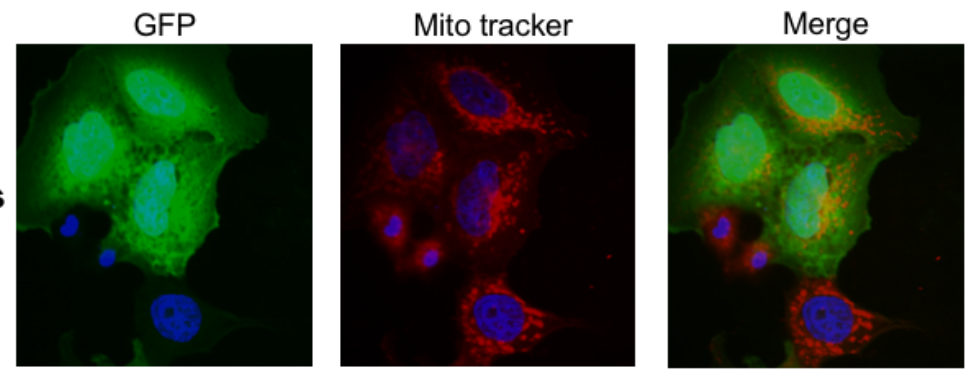

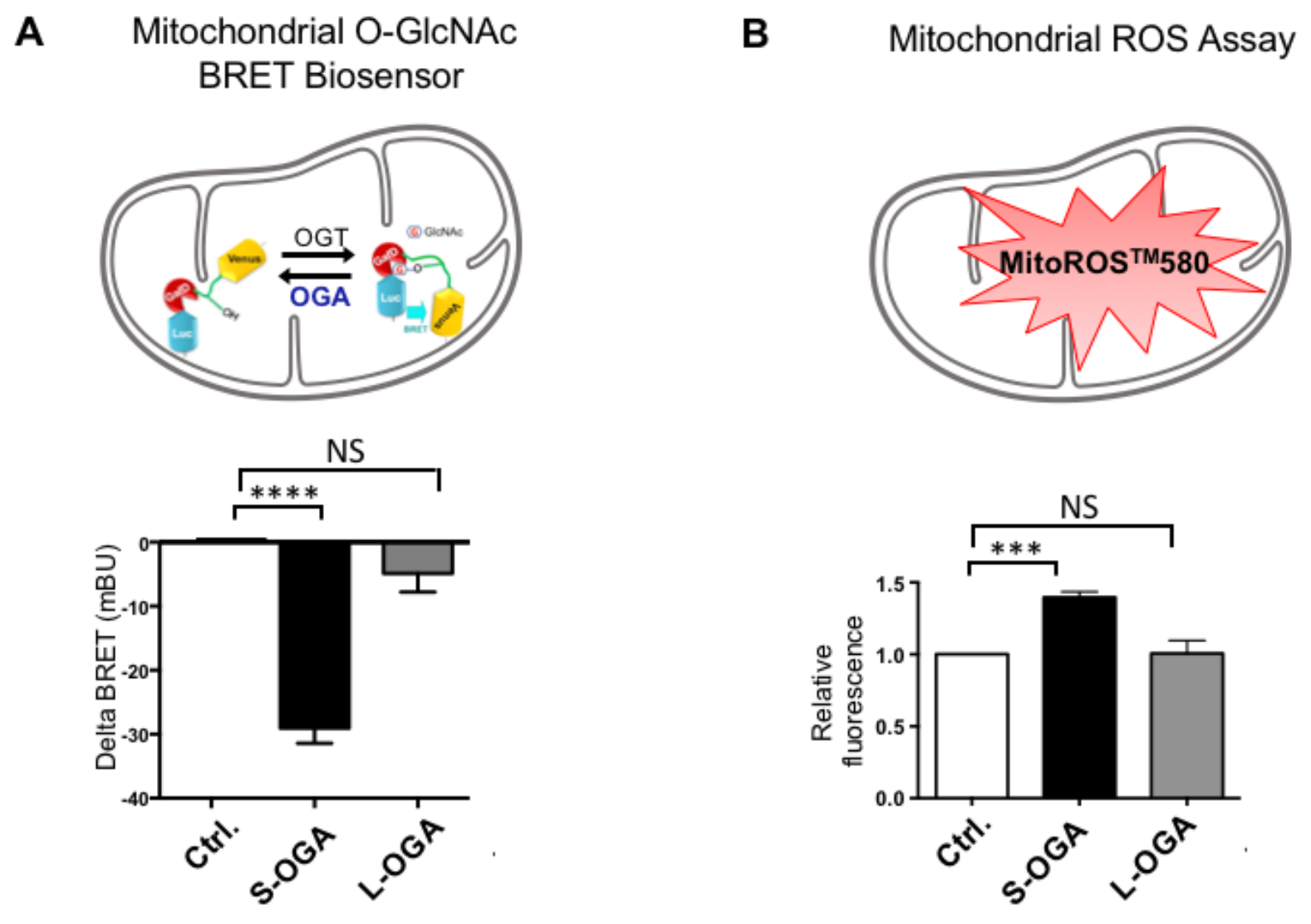

C Mitochondrial $\mathrm{H}_{2} \mathrm{O}_{2}$

HypPer7 Biosensor
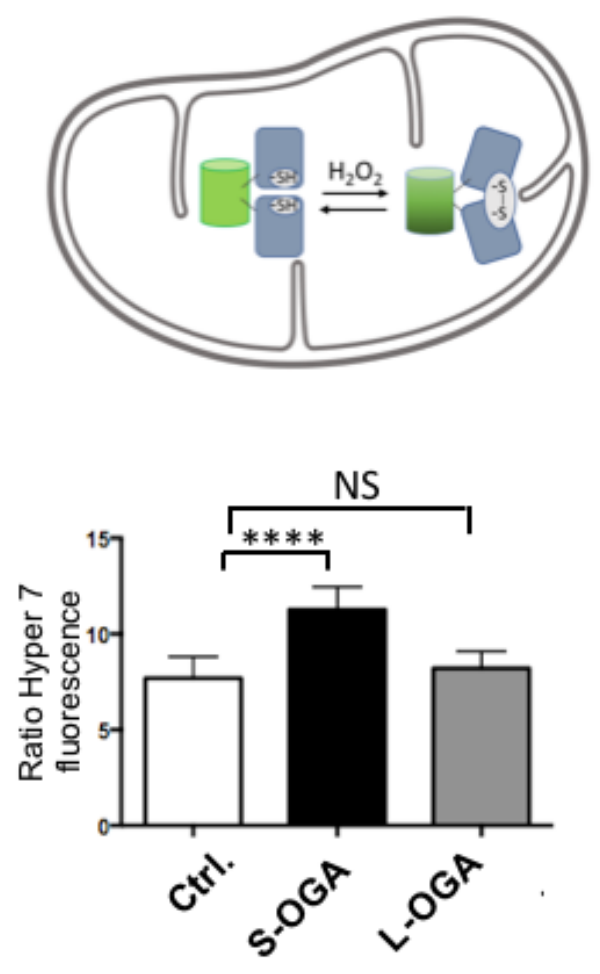
A

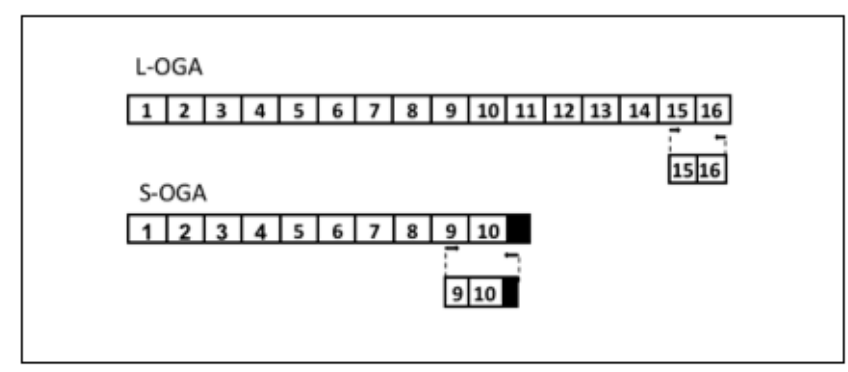

B

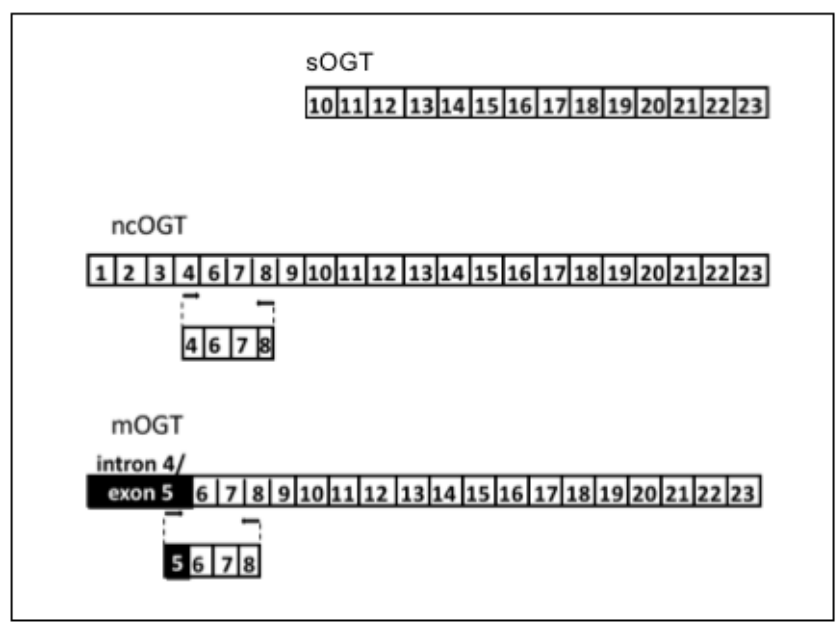

C
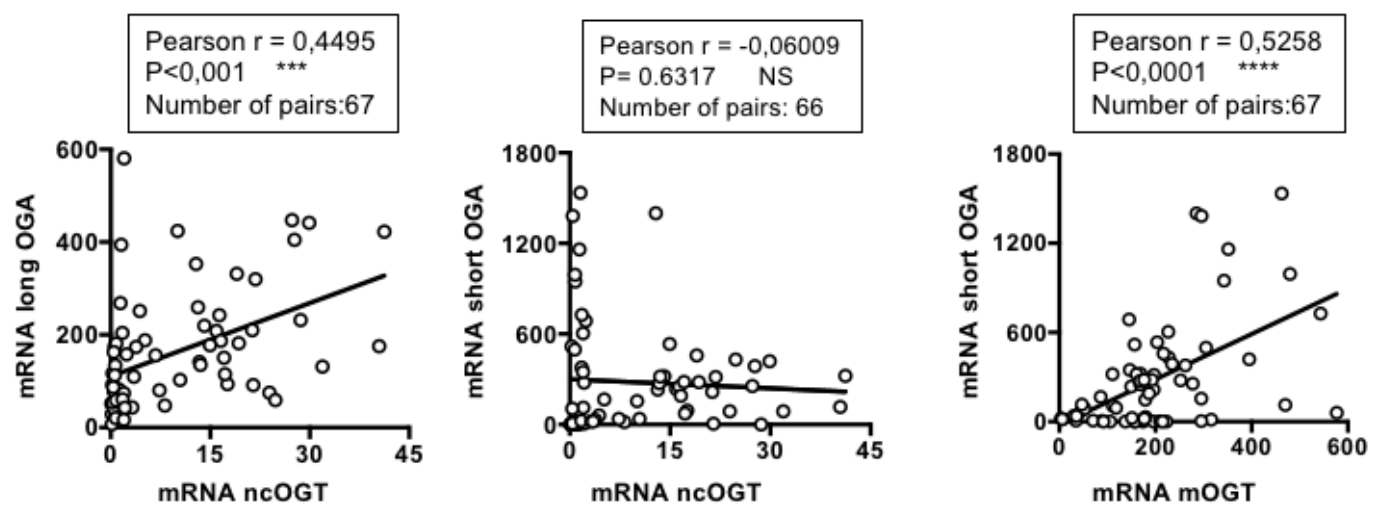
A

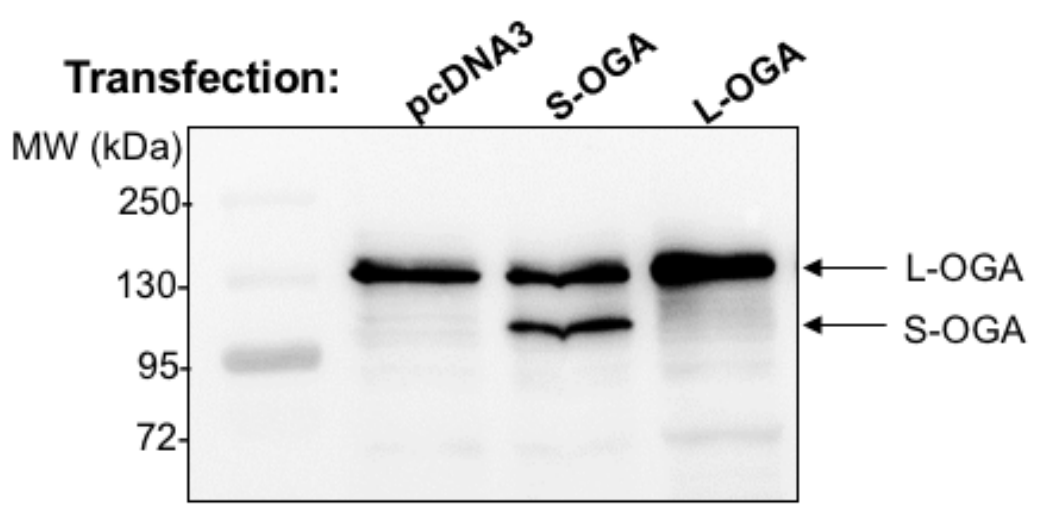

B

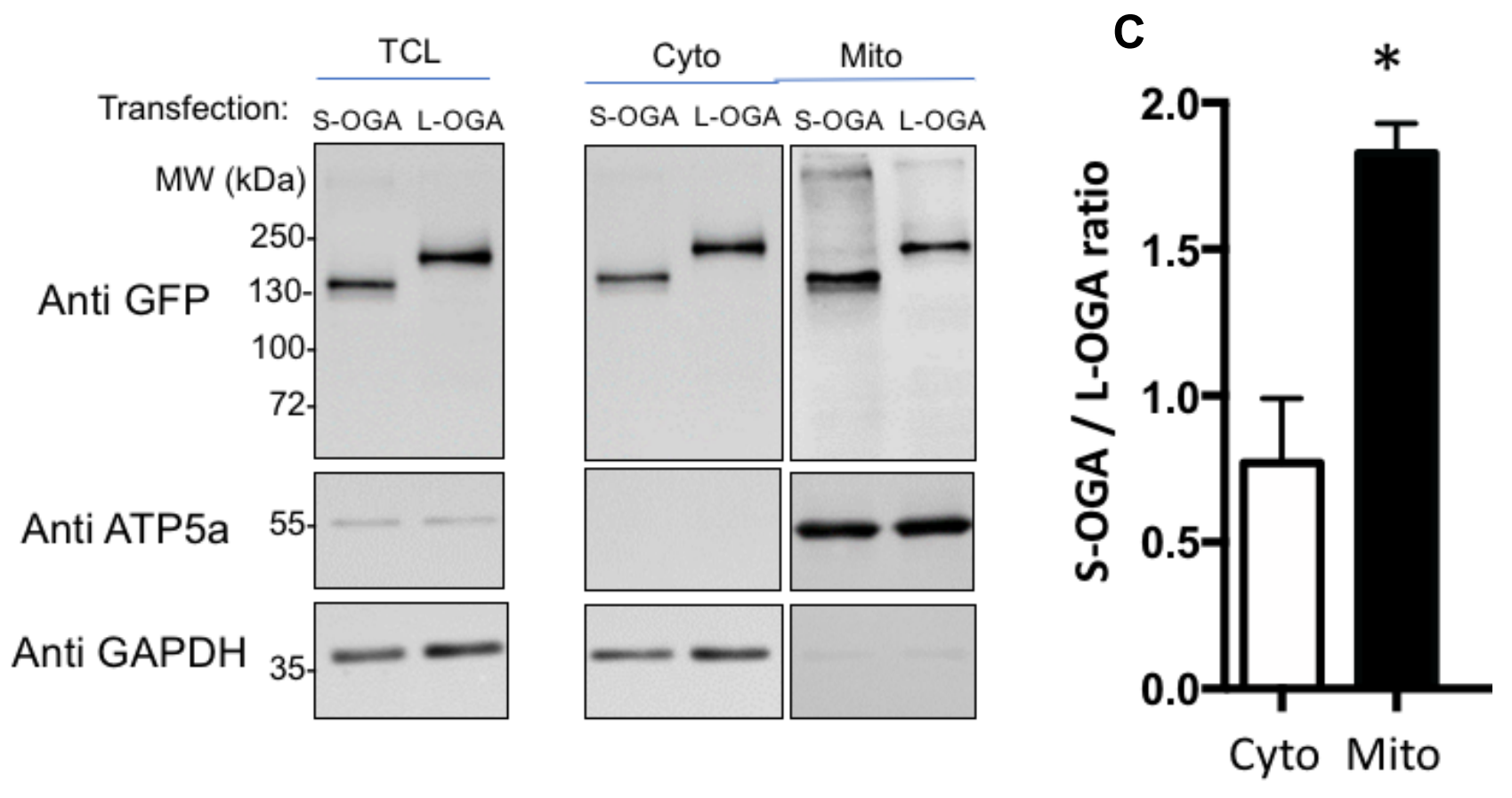

D
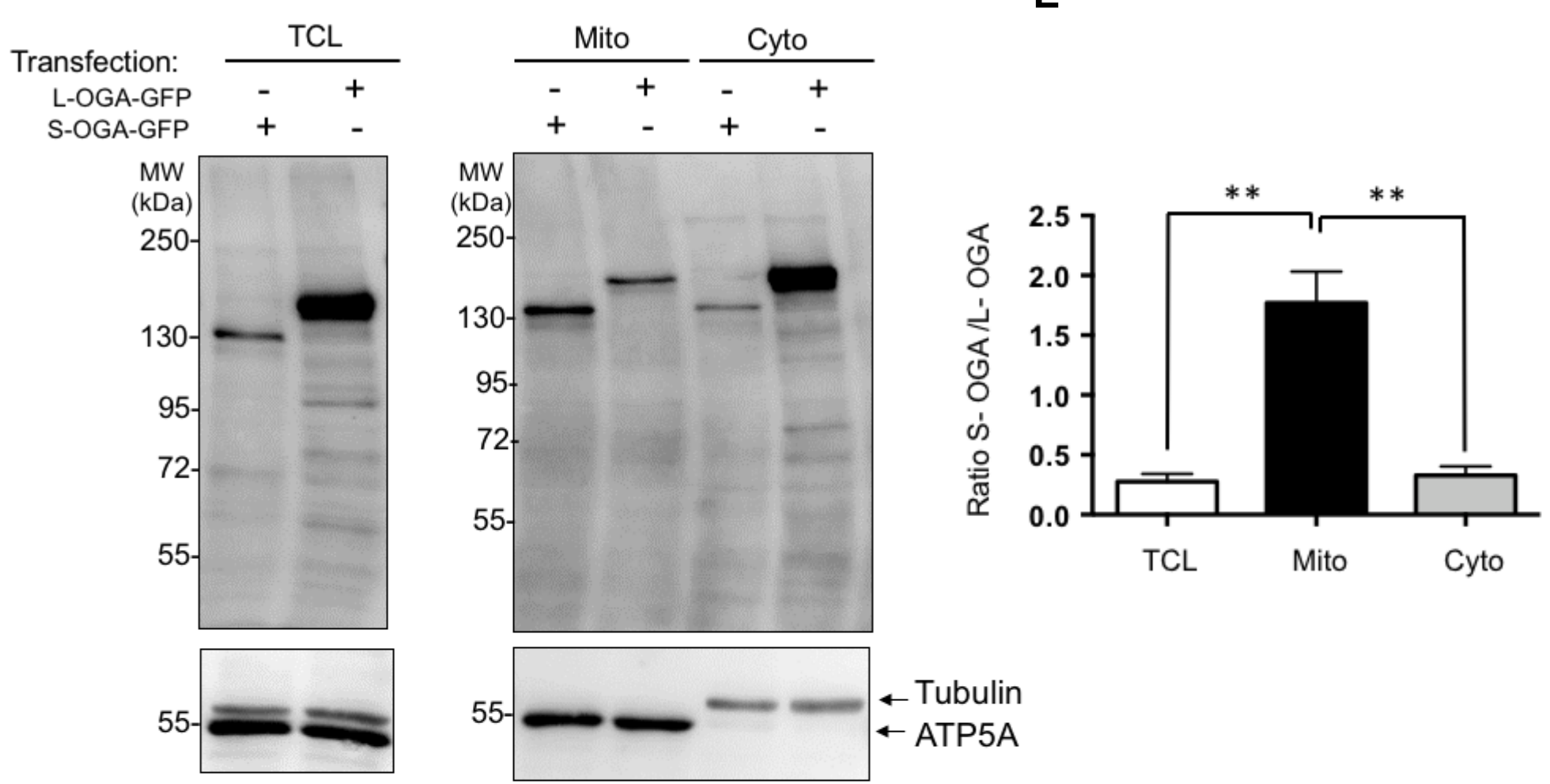
S-OGA Intron-derived peptide sequence

RCTRNNLFSSNILSL STOP

Human OGA

RCTRNNLFSSNILSL STOP

RCTRNNLFSSNILSL STOP

RCTRNNLFSSNILSL STOP

RCTRNNLFSSNILSL STOP

RCTRNNLFSSNILSLKKKII PQGEKHLGNWHFYKSFPFFA STOP

RCTRNNLFSSDILSLKKKKIIP STOP

RCTRNNLFSSDILSPKNEKKLSHREKNI STOP

RCTRNNLFSDILSLKRKKKLSHREKNVYEISEFPTVFKFLA STOP

RCTRNNLFSDILSLKRKKNYPTGRKTFRELVNFQLCLNSLPRFQRCSCCYRLEVQASVNITSTLIFLPWANSA STOP

RCTSSSLLSSIYIVL STOP

RCYGSSSGFEQTVVLSVSIAKGKTLKK STOP

Gorilla OGA

Orangutan OGA

Chimpanzee OGA

Macaca mulatta OGA

Marmoset OGA

Pig OGA

Dog OGA

Goat OGA

Cow OGA

Mouse OGA

Rat OGA 


\section{A The mitochondrial O-GIcNAc BRET Biosensor}

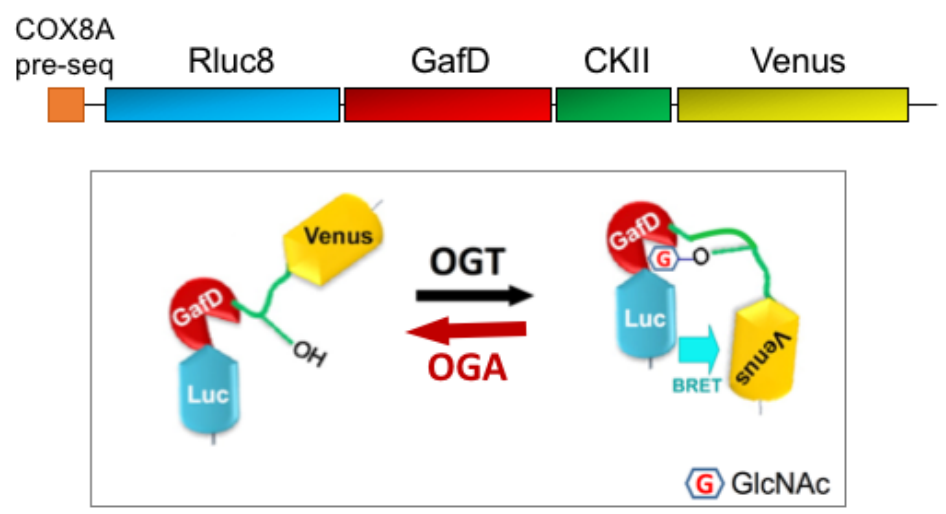

B

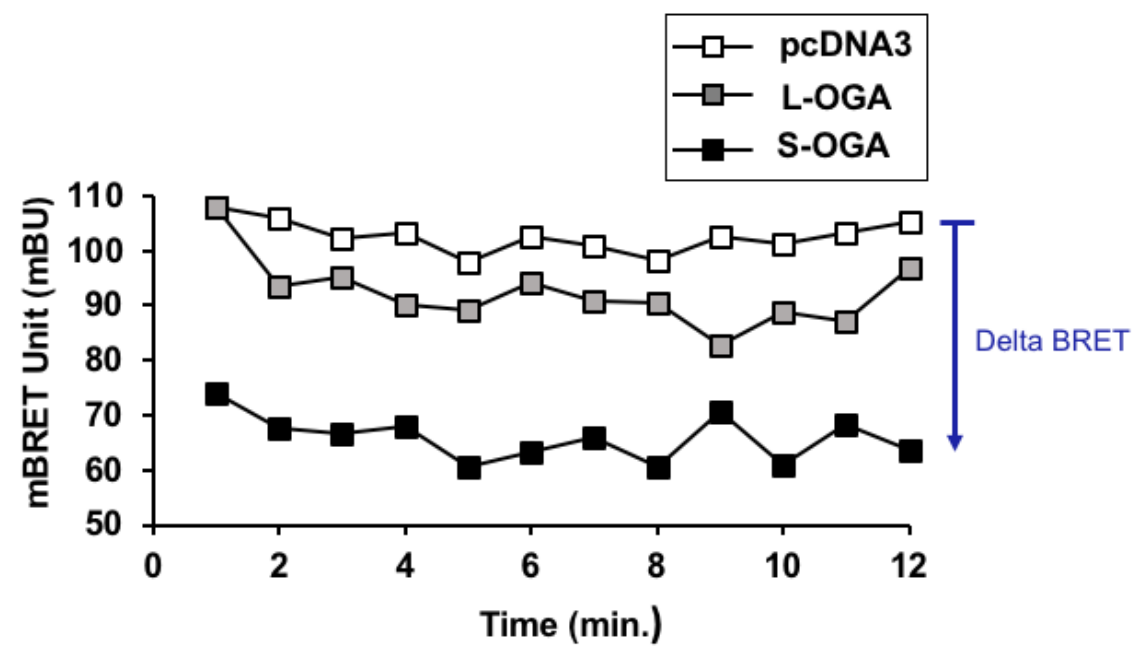

